\title{
Circadian control of P-glycoprotein in Hepa1-6 hepatocarcinoma tumor cells and Hepa1-6 tumor-bearing mice
}

\author{
Narin Ozturk ${ }^{1}$, Sandrine Dulong ${ }^{2}$, Xiao-Mei Li ${ }^{2}$, Francis Levi ${ }^{3}$, Alper Okyar ${ }^{1}$ \\ ${ }^{I}$ Department of Pharmacology, Faculty of Pharmacy, Istanbul University, Turkey, ${ }^{2}$ INSERM UMRS 935 Modeles de \\ cellules souches malignes et therapeutiques, Campus CNRS, Villejuif-Cedex, France, ${ }^{3}$ Warwick Systems Biology Centre, \\ Warwick Medical School, Cancer Chronotherapy Unit, Coventry CV47AL, UK
}

Circadian clock largely modifies efficacy and toxicity of many anticancer agents. An efflux transporter P-glycoprotein (encoded by abcb1a/abcb1b genes in rodents) is a well-known mechanism causing multidrug resistance in cancer patients by extruding anticancer drugs out of tumor cells and its expression is under the control of circadian clock. We aimed to investigate the circadian clock control of P-glycoprotein in Hepa1-6 hepatocarcinoma tumor cells in vitro and Hepa1-6 tumor-bearing mice in vivo. Bioluminescence oscillatory profile reflecting core clock gene Per2 expression was determined by Real-Time gene expression monitoring device Lumicycle32 in vitro in synchronized mouse Per2:Luc Hepa1-6 hepatocarcinoma cells obtained following stable introduction of luciferase reporter gene under the control of Per2 promoter in Hepa1-6. Abcb1a/abcb1b expression rhythms were estimated using qRT-PCR in dexamethasonesynchronized Hepa1-6 cells at 40\% and 100\% confluence sampled every 3h during 48h. Hepa1-6 tumor cells were subcutaneously injected into each flank of C57BL/6J male mice. 11 days after injection, tumors were palpable (early stage) and reached a size of $2000 \mathrm{~m} 3$ (late stage) after 23 days. Tumor tissues were removed every $3 \mathrm{~h}$ during $24 \mathrm{~h}$ from mice at early and late stage. Per2 and abcb1a/abcb1b mRNA expression rhythms were investigated with qRT-PCR in both early and late stage of proliferation. Data were analyzed with Cosinor for rhythm detection. Real-time bioluminescence pattern of Per2 revealed circadian rhythm in proliferative (Period=24h; $\mathrm{p}<0.001)$ and confluent states (Period=25h; p <0.001) in Per2:Luc Hepa1-6 cells. Abcb1a and abcb1b were expressed rhythmically along 48h in Per2:Luc Hepa1-6 cells at $40 \%$ confluence (Period $=24 \mathrm{~h} ; \mathrm{p}=0.01$ and $\mathrm{p}<0.001$ ) but not at $100 \%$ confluence ( $\mathrm{Period}=25 \mathrm{~h} ; \mathrm{p}=0.70$ and $\mathrm{p}=0.76)$. Per2 expression was not rhythmic in hepatocarcinoma cells of tumor-bearing mice in both early $(\mathrm{p}=0.08)$ and late-stage of tumor progression $(\mathrm{p}=0.36)$. Abcbla and $\mathrm{abcb} 1 \mathrm{~b}$ were expressed rhythmically along $24 \mathrm{~h}$ in Hepa1-6 tumor bearing mice in early-stage of tumor progression (Period $=24 \mathrm{~h} ; \mathrm{p}=0.03$ and $\mathrm{p}=0.02$, respectively) but no rhythm was observed in the late-stage of tumor for abcb1a and abcb1b. We provided stage-specific expression profiles of core-clock gene Per2 and P-glycoprotein genes abcb1a/abcb1b, suggesting that disturbed circadian clock gene expression and disruption of circadian rhythms correlate with tumor development and progression. 